\title{
APPLICATIONS OF MODEL PREDICTIVE CONTROLLERS IN A SUGAR FACTORY
}

\author{
Carlos Bordons and Eduardo F. Camacho
}

\author{
Dpto. Ingeniería de Sistemas y Automática, Universidad de \\ Sevilla, Spain \\ e-mails: \{bordons, eduardo\}@cartuja.us.es
}

\begin{abstract}
This paper presents two applications of Model Predictive Control in a sugar factory: temperature control in the diffusion process and density control in the wastewater treatment plant. The implementation is done using a Generalized Predictive Controller (GPC) designed for a wide class of industrial process, with the same computational requirements as a PID routine and embedded in the existing control system. The processes have in common the existence of long and uncertain dead times, therefore the original GPC algorithm is improved by the use of the T polynomial, which increases the stability robustness by filtering the predictions.
\end{abstract}

Copyright $\odot 2000$ IFAC

Keywords: Predictive control, Generalized predictive control, Robustness, Process control, Food processing.

\section{INTRODUCTION}

This paper shows two applications of GPC in a sugar refinery. The implementation was carried out by the authors in collaboration with the firm PROCISA. The refinery is located in Peñafiel (Valladolid, Spain) and belongs to Ebro Agricolas. The controllers run in a ORSI Integral Cube Control System, where the GPC has been included as a library routine which can be incorporated in a control system as easily as the built-in PID routine.

There are many applications of predictive control successfully in use at the present time (Qin and Badgwell, 1997), not only in the process industry but also applications to the control of a diversity of processes (Richalet, 1993), (Richalet et al., 1978). MPC is particularly attractive to staff with only a limited knowledge of control, because the concepts are very intuitive, and it can be used to control a great variety of processes, from those with relatively simple dynamics to other more complex ones.

The Generalized Predictive Control (GPC) method proposed by Clarke et al. (Clarke et al., 1987) is a reasonable representative of this family and has become one of the most popular MPC methods. As is well known, the basic idea of GPC is to calculate a sequence of future control signals in such a way that it minimizes a multistage cost function defined over a prediction horizon. GPC provides an explicit solution (in the absence of constraints), it can deal with unstable and non-minimum phase plants and incorporates the concept of control horizon as well as the consideration of weighting of control increments in the cost function.

A Generalized Predictive Controller results in a linear control law which is easy to implement once the controller parameters are known. The derivation of the GPC parameters requires, however, some mathematical complexities, which are difficult to solve in some industrial controllers. The industrial application of GPC in small control systems in industry has some difficulties that must be overcome. Apart from needing low computational requirements, it must be accepted by the plant operators. First, the tuning procedure must be simple enough, so that a GPC can be tuned as easily as a PID, and second, the controller must be 
robust, that is, it must behave well in the presence of the inevitable modelling errors.

The paper is organised as follows: Section 2 presents the adaptation of the standard GPC algorithm to a wide class of industrial processes in order to reduce calculations and improve robustness. Next section is dedicated to temperature control in the diffusion process, showing the commisioning of the controller as well as some operating results. Section 4 shows the application in the wastewater treatment plant and finally the conclusions of the work are presented in section 5 .

\section{PRECOMPUTED GPC}

This paper uses a formulation of Generalized Predictive Control (GPC), easy to implement and tune, that is valid for the majority of industrial processes (Camacho and Bordons, 1999), (Bordons and Camacho, 1998). The method makes use of the fact that a generalized predictive controller results in a linear control law that can be described with few parameters. The controller is valid for a wide class of processes in industry and a set of simple functions relating the controller parameters to the process parameters has been obtained. With this set of functions either a fixed or a selftuning GPC can be implemented in a straightforward manner.

Most processes in industry are high order systems that are not suitable for control purposes, but in general it is possible to approximate the behaviour of such high order processes with a simplified model consisting of a first order process combined with a dead time element (Deshpande and Ash, 1981). This model is widely used in industry to describe the dynamics of many processes, as shown by the popularity of the reaction curve method and the open loop Ziegler-Nichols PID tuning rules. Obviously better approximations could be obtained by using higher order models, but this would require identification packages which are not normally available in industry.

When the dead time $\tau_{d}$ is an integer multiple of the sampling time $T\left(\tau_{d}=d T\right)$, the corresponding discrete transfer function has the form:

$$
G\left(z^{-1}\right)=\frac{b z^{-1}}{1-a z^{-1}} z^{-d}
$$

where discrete parameters $a, b$ and $d$ can easily be derived from the continuous parameters by discretization of the continuous transfer function, resulting in the following expressions:

$$
a=e^{-\frac{T}{\tau}} \quad b=K(1-a) \quad d=\frac{\tau_{d}}{T}
$$

Therefore the CARIMA model used for the prediction when the noise polynomial is chosen equal to one is:

$$
\left(1-a z^{-1}\right) y(t)=b z^{-1} u(t-d)+\frac{1}{\triangle} \xi(t)
$$

The predictions along the horizon from $t+d+1$ to $t+d+N$ can be calculated by means of the following equation:

$$
\begin{gathered}
\hat{y}(t+d+j \mid t)=(1+a) \hat{y}(t+d+j-1 \mid t)- \\
-a \hat{y}(t+d+j-2 \mid t)+b \Delta u(t+j-1)
\end{gathered}
$$

The plant parameters are used to compute the controller coefficients $\left(l_{y 1}, l_{y 2}, l_{r 1}\right)$ as described in (Bordons and Camacho, 1998). These coefficients are precalculated as a function of the system pole (a) and the control weighting factor $(\lambda)$ with horizons $N_{1}=d+1, N_{2}=d+N, N_{u}=N$, $N=15$. The values $\hat{y}(t+d \mid t), \hat{y}(t+d-1 \mid t)$ are obtained by the use of the prediction which basically consists of a model of the plant which is projected towards the future with the values of past inputs and outputs and only requires straightforward computation. The control law is given by:

$$
\begin{gathered}
\Delta u(t)=l_{y 1} \hat{y}(t+d \mid t)+l_{y 2} \hat{y}(t+d-1 \mid t)+ \\
+l_{r 1} r(t)
\end{gathered}
$$

The control algorithm reduces to:

1. Compute $k_{j i}$ as functions of $\lambda$.

2. Make $l_{y i}=k_{1 i}+k_{2 i} \frac{\hat{a}}{k_{3 i}-\dot{a}}$ for $i=1,2$

and $l_{r 1}=-l_{y 1}-l_{y 2}$

3. Compute $\hat{y}(t+d \mid t)$ and $\hat{y}(t+d-1 \mid t)$ using equation (3) recursively.

4. Compute control signal $u(t)$ with:

$\Delta u(t)=l_{y 1} \hat{y}(t+d \mid t)+l_{y 2} \hat{y}(t+d-1 \mid t)+$ $+l_{r 1} r(t)$

It can be seen that the algorithm is really simple and can be easily included in any commercial control system without complex calculation requirements. This algorithm has been successfully tested in some experimental plants. However, it has also been shown (Camacho and Bordons, 1995) that although it is rather robust to gain and time constant uncertainties, it has small robustness to deadtime uncertainties, that are commonly found in real plants. That is why the algorithm must be modified to consider this circumstances, since the processes to be controlled present that kind of uncertainty (as will be seen later).

The stability robustness of GPC can be improved with the use of an observer polynomial, the socalled $T\left(z^{-1}\right)$ polynomial. In (Clarke and Mo- 
htadi, 1989) a reformulation of the standard GPC algorithm including this polynomial can be found. In order to do this, the CARIMA model is expressed in the form:

$$
A\left(z^{-1}\right) y(t)=B\left(z^{-1}\right) u(t-1)+\frac{T\left(z^{-1}\right)}{\triangle} \xi(t)
$$

Up to now the $T\left(z^{-1}\right)$ has been considered equal to 1 , describing the most common disturbances but it can also be considered as a design parameter. In consequence the predictions will not be optimal but on the other hand robustness in the face of uncertainties can be achieved, in a similar interpretation as that used by Ljung (Ljung, 1987). Then this polynomial can be considered as a prefilter as well as an observer. The effective use of observers is known to play an essential role in the robust realization of predictive controllers (see (Clarke and Mohtadi, 1989) for the effect of prefiltering on robustness).

This polynomial can be easily added to the proposed formulation, computing the prediction with the values of inputs and outputs filtered by $T\left(z^{-1}\right)$. Then, the predictor works with $y^{f}(t)=$ $\frac{y(t)}{T\left(z^{-1}\right)}$ and $u^{f}(t)=\frac{u(t)}{T\left(z^{-1}\right)}$. The actual prediction for the control law is computed as $\hat{y}(t+d)=$ $T\left(z^{-1}\right) \hat{y}^{f}(t+d)$.

The correct choice of the $T$ polynomial is a problem that has not completely been solved, although its effect on the robustness of the closed loop system has been analysed in several papers (Clarke $e t$ al., 1987), (Clarke and Mohtadi, 1989), (Robinson and Clarke, 1991), (Yoon and Clarke, 1995). In this application, $T$ is made equal to $A\left(z^{-1}\right)(1-$ $\beta z^{-1}$ ), being $\beta$ a value close to the system pole, as suggested in (Yoon and Clarke, 1995).

\section{TEMPERATURE CONTROL IN THE DIFUSSION PROCESS}

The factory produces sugar from sugar-beet by means of a series of processes such as precipitation, cristalization, etc. The process that is controlled in this section is the temperature control of the descummed juice in the diffusion.

In order to extract the sugar from the beet it is necessary to dilute the saccharose contained in the tuber tissue in water in order to form a juice from which sugar for consumption is obtained. The juice is obtained in a process known as diffusion. Once the beet has been cut into pieces to increase the interchangable surface, it enters into the macerator (which revolves at a velocity of 1 r.p.m.) where it is mixed with part of the juice coming from the diffusion process (see figure 1). Part of the juice inside the macerator is recirculated in order to be heated by means of steam and in

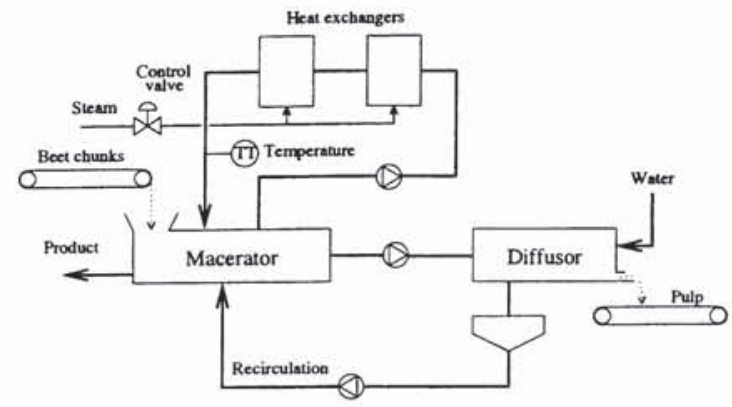

Fig. 1. Diffusion Process

this way it maintains the appropriate temperature for maceration. The juice from the maceration process passes into the diffusor (a slowly revolving pipe $25 \mathrm{~m}$ long and with a diameter of $6 \mathrm{~m}$ ) where it is mixed with water and all the available sugar content is extracted, leaving the pulp as a sub-product. The juice coming out of the diffusor is recirculated to the macerator, from which the juice already prepared is extracted for the next process.

In order for the diffusor to work correctly it is necessary to supply thermal energy to the juice during maceration. In order to obtain this objective, part of the juice from the the macerator (about $150 \mathrm{~m}^{3} / \mathrm{h}$ ) is made to recirculate through a battery of exchangers; within these the steam proceeding from the general services of the factory provides the heat needed to obtain optimum maceration. Therefore the controller must adjust the steam valve $(u)$ in order to achieve a determined return temperature to the macerator $(y)$.

The system response is seriously disturbed by changes in the steam pressure, which are frequent because the steam used in the exchangers has to be shared with other processes which can function in a non-continuous manner.

The process is basically a thermal exchange between the steam and the juice in the pipes of the exchanger, with overdamped behaviour and delay associated to the transportation time of the juice through pipes about 200 meters long. These considerations, together with the observation of the development of the system in certain situations, justify the use of a first order model with delay.

A model was identified by its step response measuring the temperature when the valve is open. The values of gain, time constant and delay can easily be obtained from the response:

$$
K=0.1905 \frac{{ }^{\circ} \mathrm{C}}{\%} \quad \tau=5 \mathrm{~min} \quad \tau_{d}=1 \mathrm{~min} 45 \mathrm{~s}
$$

However, it is seen that the system reacts differently when heated to when cooled, the delay being quite a lot greater in the first case. A similar test closing the valve again provides values of 
$K=0.15 \tau=5 \min 20 \mathrm{~s} \quad \tau_{d}=4 \min 50 \mathrm{~s}$

Although an adaptive strategy could be used (with the consequent computational cost), a fixed parameter controller was employed, showing, at the same time, the robustness of the method when using the T-polynomial in presence of modelling errors. The error in the delay, which is the most dangerous, appears in this case. The following values of the model were chosen for this:

$$
K=0.18 \quad \tau=300 \mathrm{~s} \quad \tau_{d}=190 \mathrm{~s}
$$

and sampling time of $\mathrm{T}=60 \mathrm{~s}$.

It should be noticed that there are great variations in the delay (that produced on heating is about three times greater than that on cooling), due to which it is necessary to introduce the filter $T\left(z^{-1}\right)$ in order to increase the robustness.

With the nominal model chosen, the discrete parameters of the process model are given by:

$$
a=0.8187 \quad b=0.0326 \quad d=3
$$

The controller coefficients can be computed (see (Bordons and Camacho, 1998)) calculating $k_{j i}(\lambda)$ and then $l_{y 1}, l_{y 2}$ and $l_{r 1}$ as seen in the previous section.

As the system pole is in 0.8187 , if a value of $\lambda$ equal to 0.2 is chosen, the controller coefficients are

$$
l_{y 1}=-4.2914 \quad l_{y 2}=2.4165 \quad l_{r 1}=1.8749
$$

The behaviour of the controller rejecting the disturbances (brusque variations in the steam pressure and load changes) can be seen in figure 2 .

The controller interface allows the process parameters to be changed on line. The model is tuned by the operator as soon as a discrepancy between the actual and the predicted outputs (that appear on the screen) is detected.

Following many operational days the operators themselves concluded that a satisfactory model was given by:

$$
K=0.25 \quad \tau=250 \mathrm{~s} \quad \tau_{d}=220 \mathrm{~s}
$$

with a control weighting factor $\lambda=0.1$, a sampling time of 50 seconds and robust filter of $T\left(z^{-1}\right)=A\left(z^{-1}\right)\left(1-0.8 a z^{-1}\right)$, being $a$ the discrete pole.

Notice that these results have not been compared to those of using the controller without the $T$ polynomial. The reason for this is that removing the polynomial made the closed loop unstable (due to the big dead time uncertainty). As it is a real application, the filter had to be included in
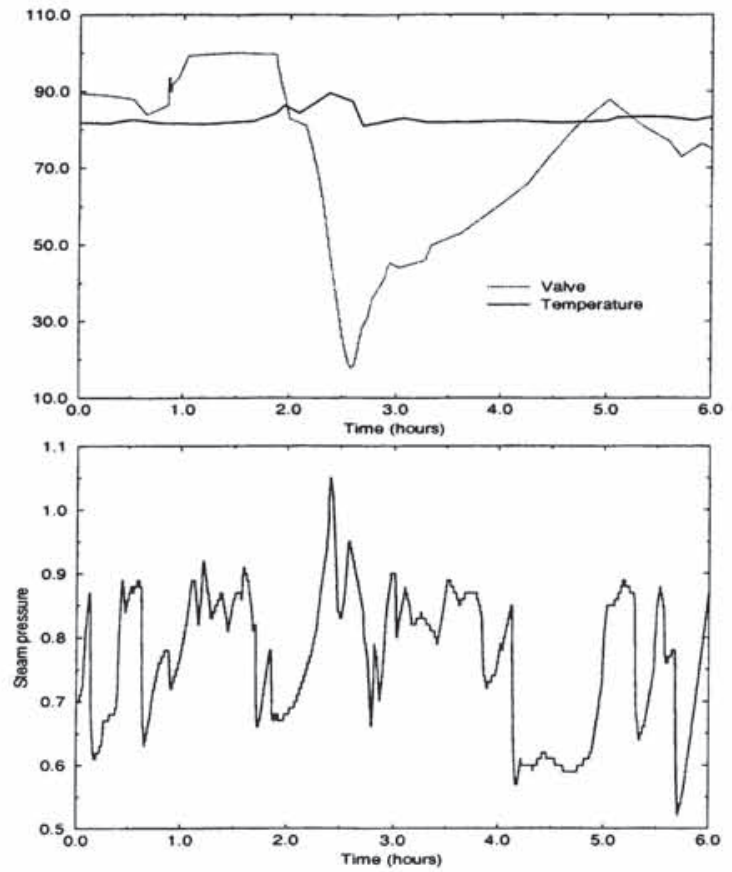

Fig. 2. System response in the presence of external disturbances

the controller when the output started to show a dangerous behaviour.

\section{DENSITY CONTROL IN WASTE WATER TREATMENT}

The factory handles a large quantity of water; water is mainly necessary for producing steam in the boilers, for the diffusion process and for washing the sugar-beet. The water is not actually consumed so although used in various processes it is recoverable. The objective is that the factory should be completely self-sufficient with regard to water consumption.

One of the processes which consumes most water is washing the beet. The raw material is washed using clean water to eliminate any remaining soil which logically must not enter into the sugar producing process. This operation results in large amounts of dirty water (sludge) being produced which must be recycled.

The recovery of clean water from this sludge is achieved by using the process shown in figure 3 . A separating tank is used where sedimentation by gravity takes place, the water stays on the surface and the sludge sinks to the bottom. This separating tank is an open container with a diameter of 40 meters and an average depth of 5 meters. It is fitted with a blade which revolves at $0.05 \mathrm{rpm}$ and which ejects the thicker remains into a $35 \mathrm{~m}^{3}$ homogenizer cylinder from which it is pumped into the centrifuge which revolving at great speed separates the water from the soil by 


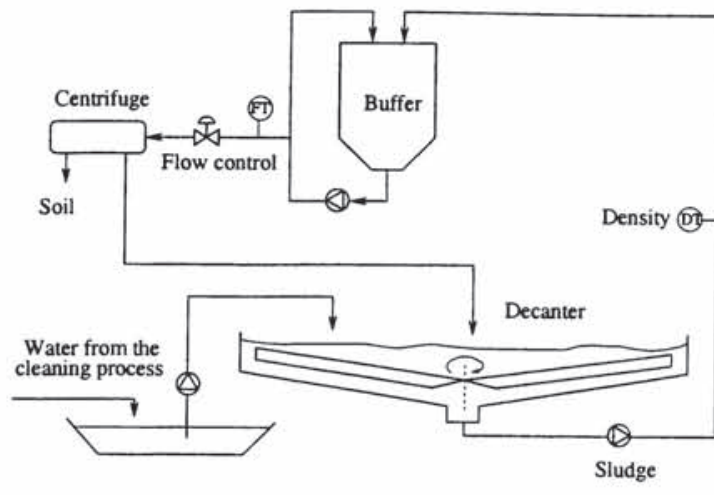

Fig. 3. Water Recovery Process

centrifugation. The water thus obtained returns to the separating tank from where the usable water is again drawn out.

The centrifuge works correctly for sludge flows of between 14 and $22 \mathrm{~m}^{3} / \mathrm{h}$ and a density which should be between 1100 and $1200 \mathrm{~g} / \mathrm{l}$. This density is the basic variable to be controlled as it must remain within this range to ensure the correct working of all the installation. This density depends upon the water recycled from the centrifuge to the separating tank and therefore on the sludge flow that circulates from the cylinder to the centrifuge.

It is difficult to model the relationship between the recirculation flow and the density, because various factors come into play and there is no clear relationship between said variables. However, it does seem clear that an increase in the flow causes more water to be recirculated and thus the density of the sludge to be decreased; a decrease of density has the opposite effect. An exhaustive modelling is rather complicated so a first order system with delay will be used to try to approximate the response.

An added problem is that the flow measurements are not very reliable due to the particles in suspension. Furthermore there are continuous blockages in the control valve which cause brusque alterations in the flow. On the other hand, because of the movement of the blades in the separating tank which stir the mud that will be pumped towards the centrifuge, the density measurements are not uniform because they keep falling like lumps of soil everytime the blade passes over the drain. Therefore, a treatment of the density meter signal is needed.

As the recirculation flow suffers frequent disturbances due to the presence of discontinuities in the fluid composition, it is necessary to keep it controlled with another regulator. The control strategy is, therefore, going to be a cascade control, the density control acting on the remote setpoint of the local controller of the flow of the centrifuge (slave controller), see figure 4. This local control

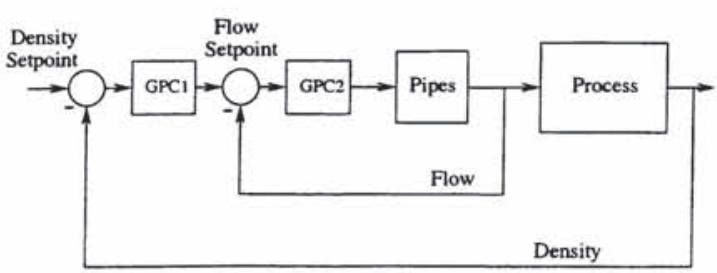

Fig. 4. Cascade controllers

can be carried out using either a PI controller or a GPC indistinctively. The dynamics of this loop (a simple flow loop) does not justify itself the use of a GPC instead of a PI, but as the GPC routine is already installed in the control system and the computation time is similar in both cases, this loop was also used as a testbed for the controller. Besides, the tuning of the GPC is done automatically every time the system pole is updated.

Following an initial analysis it is observed that the characteristic time of the system response is in the order of hours. In order to obtain a model, a step is provoked at the input, obtaining from the data a dead time of 2.6 hours, a time constant of 1 hour and a gain of $-25 \frac{\mathrm{g} / \mathrm{l}}{\mathrm{m}^{3} / \mathrm{h}}$.

The control was started up using filtering with the T-polynomial made equal to $A\left(z^{-1}\right)\left(1-\beta z^{-1}\right)$, with $\beta=0.9$. The control effort $\lambda$ was chosen equal to 0.1 . The local flow controller had previously been adjusted by the step response procedure obtaining $K=2.5, \tau=4 \mathrm{~s}$ and $\tau_{d}=10 \mathrm{~s}$ (notice the clear difference in the dynamics between the outer and inner loops).

With the nominal model chosen for the density loop and with a sampling time of 12 minutes, the discrete parameters of the process model are given by:

$$
a=0.8187 \quad b=-0.4531 \quad d=13
$$

Using the procedure described in (Bordons and Camacho, 1998), as the system pole is in 0.8187 , if a value of $\lambda$ equal to 0.1 is chosen, the controller coefficients are

$$
l_{y 1}=-4.7454 \quad l_{y 2}=2.5930 \quad l_{r 1}=2.1524
$$

A similar procedure is used for the obtention of the fast flow controller, with values:

$$
l_{y 1}=-3.2448 \quad l_{y 2}=2.1294 \quad l_{r 1}=1.1154
$$

The control system operation is shown in the following diagrams. Note the time scale, where the slowness in the density evolution can be seen, two hours being necessary in order to carry out a change in the setpoint (see figure 5). Notice that the output shows an oscillatory signal added to the mean value. These oscillations are density changes due to the effect of the blades which 


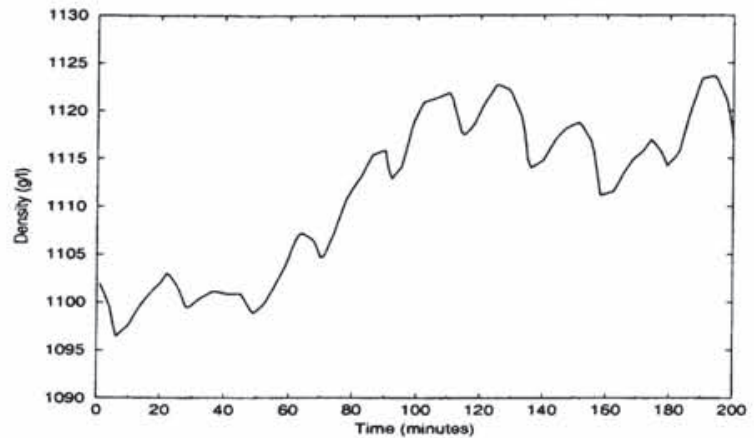

Fig. 5. Setpoint change



Fig. 6. Disturbance rejection in the density loop stir the mud in the decanter, so the density measurements show this response because they keep falling like lumps of soil everytime the blade passes over the drain.

Note the importance of introducing the $\mathrm{T}$ polynomial, given that the dead time obtained experimentally is not very precise and that also other tests showed that its value varied substantially from one situation to another.

The main density loop objective is to reject disturbances. Figure 6 shows the behaviour of the controller during eight hours keeping the density at the right value of $1090 \mathrm{~g} / \mathrm{l}$.

\section{CONCLUSIONS}

Two applications GPC in a sugar factory have been presented. The control law was extremely simple to compute and the tuning was straightforward because of the low order model used. The original GPC algorithm was improved by the use of the $\mathrm{T}$ polynomial to increase the stability robustness, since model uncertainties appeared when working at different operating points. It should be emphasised that the controllers worked satisfactorily and without interruption until the end of the year's campaign, being handled without difficulty by the plant operators.

The application shown here combines the power of predictive control with the simplicity and ease of use of the traditional controllers commonly found in industry, showing how a GPC can be easily used in clasical control structures like cascade control in the same way as PIDs.

\section{ACKNOWLEDGMENTS}

The authors would like to acknowledge Juan Hermida from PROCISA by his interest and support in the development of the controller and the people from Ebro Agricolas by the facilities for testing the controller in the plant. The financial support of CICYT by projects TAP 96-0884 and TAP 980541 and EC by project 1FD97-0836 is gratefully appreciated.

\section{REFERENCES}

Bordons, C. and E.F. Camacho (1998). Generalized Predictive Controller for a Wide Class of Industrial Process. IEEE Transaction on Control Systems Technology 6(3), 372-387.

Camacho, E.F. and C. Bordons (1995). Model Predictive Control in the Process Industry. Springer-Verlag.

Camacho, E.F. and C. Bordons (1999). Model Predictive Control. Springer-Verlag.

Clarke, D.W. and C. Mohtadi (1989). Properties of Generalized Predictive Control. Automatica 25(6), 859-875.

Clarke, D.W., C. Mohtadi and P.S. Tuffs (1987). Generalized Predictive Control. Parts I and II. Automatica 23(2), 137-160.

Deshpande, P.B. and R.H. Ash (1981). Elements of Computer Process Control. ISA.

Ljung, L. (1987). System Identification. Theory for the user. Prentice-Hall.

Qin, S.J. and T.A. Badgwell (1997). An Overview of Industrial Model Predictive Control Technology. In Chemical Process Control: Assessment and New Directions for Research. In: AIChE Symposium Series 316, 93. Jeffrey C. Kantor, Carlos E. Garcia and Brice Carnahan Eds. 232-256.

Richalet, J. (1993). Industrial Applications of Model Based Predictive Control. Automatica 29(5), 1251-1274.

Richalet, J., A. Rault, J.L. Testud and J. Papon (1978). Model Predictive Heuristic Control: Application to Industrial Processes. Automatica 14(2), 413-428.

Robinson, B.D. and D.W. Clarke (1991). Robustness effects of a prefilter in Generalized Predictive Control. Proceedings IEE, Part D 138, 2-8.

Yoon, T.W. and D.W. Clarke (1995). Observer Design in Receding-Horizon Control. International Journal of Control 2, 151-171. 